\title{
Diagnostic Usefulness of Chest Computed Radiography-Film Versus Cathode-Ray Tube Images
}

\author{
Takeo Ishigaki, Sadayuki Sakuma, Tokiko Endo, and Mitsuru Ikeda
}

\begin{abstract}
Seventy-one plain chest images obtained by computed radiography (CR) with an imaging plate were interpreted on film and two kinds of cathode-ray tube (CRT) monitors installed separately at two facilities $(1,024 \times 1,536$ pixels, 8 bits, and 1,024 $\times 1,280$ pixels, 10 bits) by 20 radiologists and four chest internists. The clinical categories of these 71 cases included pulmonary nodules and interstitial abnormalities. Image reading sessions were held over a total of 4 days, ie, 2 days and then another 2 days, 3 weeks later. Twenty-four observers formed four groups with six members each. Two groups read either films or CRT images at one of the two facilities. In the second experiment, 26 of 71 images were compressed at 10:1, 19 of 71 were compressed at 20:1, and 26 were not compressed. Analyses of the areas under the receiveroperating characteristic curves showed no significant differences in detection of pulmonary abnormalities between film and CRT. In detecting interstitial pulmonary abnormalities, film was more sensitive than CRT monitor. There were no significant differences in observers' performances between the two different kinds of CRT workstation. Subjective evaluation of image quality showed that images irreversibly compressed to the ratios of 10:1 and 20:1 were inferior to original images. Although further considerations are needed with regard to spatial resolution requirements, image processing, and image compression, the utilization of CR CRT image as a substitute for CR film image will be possible.
\end{abstract}

Copyright 1995 by W.B. Saunders Company

KEY WORDS: digital storage-phosphor imaging, computed radiography, cathode-ray tube image, soft-copy image, image compression.

$\mathbf{O}$ WING TO THE DEVELOPMENT of computed radiography (CR) with imaging plates, digitization has become practical in the field of $x$-ray imaging. ${ }^{1}$ One of the major requirements for implementation of a picture archiving and communication system (PACS) is that the images must be displayed on a cathoderay tube (CRT) monitor without loss of information. However, the quality of an image depends upon the performance of the CRT. There have been many reports on the effect of pixel size on the quality of digitized hard-copy film image and soft-copy CRT image..$^{2-7}$ In a comparison of diagnostic efficacy between screen-film images and digitized images displayed on hard copy and soft copy, some authors reported that soft-copy images were acceptable, ${ }^{8,9}$ whereas others found soft copies unacceptable especially for the detection of subtle interstitial disease and pneumothorax. ${ }^{10-12}$ In addition, previous reports have indicated the possibility of CRT diagnosis of computed radiography (CR) images as a substitute for CR film diagnosis, although it may depend on the spatial resolution of CRT monitor. ${ }^{11,13-16}$ Another problem to be considered before PACS can be realized is irreversible image compression. With the use of an improved discrete cosine transformation technique, the upper limit of the compression ratio in interpretation of CR CRT chest images was 20:1 in small-scale receiver-operating characteristic (ROC) studies. ${ }^{17}$ However, not only studies within a single institution, but also large-scale cooperative experimental studies are necessary to more thoroughly evaluate the possibilities of CR CRT diagnosis and image compression. The present study conducted a large-scale reading experiment involving multiple observers from several medical institutions to assess the diagnostic efficacy of CRT images in comparison with film images, differences between two kinds of CRT monitors, and irreversible compression of images.

\section{MATERIALS AND METHODS}

In the present study, CR images stored for a long periods of time on optical disks were displayed both on film and on two different kinds of CRT. Every image was read twice in each form, or a total of four times by 24 observers, and the results were compared.

The observers comprised a total of 24 physicians from 12 institutions. Twenty of 24 observers were diagnostic radiologists and 13 were board certified. Their level of experience as a diagnostic radiologists ranged from 3 to 25 years. Four of 24 observers were chest internists with 8 to 11 years clinical experience including interpretation of chest radiographs. They had no or limited experience with CRT monitors and interactive windowing.

Image reading sessions were held for a total of 4 days, ie,

From the Department of Radiology, Nagoya University School of Medicine, Nagoya, Japan.

Address reprint requests to Takeo Ishigaki, MD, Department of Radiology, Nagoya University School of Medicine, 65, Tsurumaicho, Showa-ku, Nagoya, 466, Japan.

Copyright $(1995$ by W.B. Saunders Company

0897-1889/95/0801-1008\$3.00/0 
Table 1. Sequence of the Facility in Each Group

\begin{tabular}{lll}
\hline & Experiment 1 & Experiment 2 \\
\hline Group A & Nagoya & Miyanodai \\
Group B & Miyanodai & Nagoya \\
Group C & Nagoya & Nagoya \\
Group D & Miyanodai & Miyanodai \\
\hline
\end{tabular}

Table 2. Clinical Categories of 71 Cases

\begin{tabular}{lc}
\multicolumn{1}{c}{ Category } & No. of Cases \\
\hline Normal & 18 \\
Interstitial disease & 11 \\
Pneumonia & 5 \\
Nodule & \\
Solitary, and below $10 \mathrm{~mm}$ & 4 \\
Multiple, and below $10 \mathrm{~mm}$ & 7 \\
Solitary, 11-20 mm & 15 \\
Solitary, 21-30 mm & 9 \\
Rib lesion & 2 \\
\hline
\end{tabular}

2 days (first experiment) and another 2 days (second experiment) 3 weeks after the first experiment at Nagoya University (NGY; Nagoya, Japan) and at the Miyanodai Technology Development Center of Fuji Photo Film Co Ltd (MYD; Kanagawa, Japan). Two different kinds of CRT workstations were used for interpretation of CR images. At NGY, six CRT workstations (20-inch monitor with 2:1 interlace mode) with a spatial resolution of $1,024 \times 1,536$ pixels and a gray-level resolution of 8 bits (TDIS-File, Toshiba Co Ltd, Tokyo, Japan) was used. At MYD, six CRT workstation (20-inch monitor with noninterlace mode) with a spatial resolution of $1,024 \times 1,280$ pixels and a gray-level of 10 bits (HI-C653, Fuji Photo Film Co. Ltd. Tokyo) were used. In both the first and second experiments, the same observers alternately viewed film images and CRT images, each for 1 day. That is, at these facilities, the 24 observers formed four groups of six members each (groups A, B, C, D). Each group read either film or CRT images displays on each day. The order of image reading was randomly assigned to each observer. In the second experiment, two groups read images at the same facilities as in the first experiments, whereas the other two groups read images at the facility other than that visited in the first experiment. The sequence of the facility in each group is shown in Table 1.

The CRT images used for reading were selected from images contributed by five institutions. A total of 71 images stored in reversibly compressed form were selected. None of the cases contained distinguishing or unique characteristics, such as an unusual foreign object or dominant pathologic finding to compensate for the short period between two reading sessions. The presence or absence of abnormality as

$\begin{gathered}\text { Table 4. Comparison of Areas Under the ROC Curves } \\
\text { Between Film and CRT in the Total of } 12 \text { Observers }\end{gathered}$
\begin{tabular}{ccccc} 
NGY Exp. 1 & NGY Exp. 2 & MYD Exp. 1 & MYD Exp. 2 \\
\hline Film $0.837(0.02)$ & $0.851(0.02)$ & $0.767(0.02)$ & $0.845(0.02)$ \\
CRT & $0.831(0.02)$ & $0.819(0.02)$ & $0.792(0.02)$ & $0.845(0.02)$ \\
\hline
\end{tabular}

Values in parentheses show standard error.

well as the visibility of abnormality were determined by unanimous agreement among three radiologists who did not participate in the ROC study. The clinical categories of the 71 images were as shown in Table 2. All image data used was collected on an optical disc and printed on a film. A pair of CR images $(7 \times 7$ inches, $1760 \times 1760$ pixels, $0.2-\mathrm{mm}$ spatial resolution, 10 bits) was printed on hard copy; one was produced with no image processing (default mode), whereas the other was processed with slight gradation and a frequency-processing mode. The images used in the first experiment comprised both film and CRT versions of the 71 uncompressed images, whereas in the second experiment, 26 of the former 71 images were compressed at 10:1, 19 of 71 were compressed at 20:1, and 26 were not compressed. The compression technique was commercially available Fuji CR mode with predictive coding for reduction of spatial redundancy. ${ }^{18}$

No reading time limit was set for either film and CRT images. Because the diagnostic accuracy obtainable by CRT reading is influenced by the illumination of the room, ${ }^{19}$ the illumination of CRT reading room was adjusted to a range of 170 to 200 lux at both facilities. In the hard-copy CR film reading process, the images were interpreted on a conventional view box equipped with a black-border mask to exclude extraneous light. CR film images were diagnosed by observing a pair of images, ie, a left-hand image and a right-hand image. In the case of CRT images, gradation processing and zooming were freely available. Observers were requested to determine the presence or absence of pulmonary abnormalities with one of five levels of confidence $(1$, definitely absent; 2 , probably absent; 3 , equivocal; 4 , probably present; 5 , definitely present) and to make an entry regarding the site of lesion on a lung field scheme. In addition, possible specific diagnosis was listed with one of three levels (definite, almost definite, or suspected). For each case, the observers also noted the subjective evaluation of the image quality as a chest radiograph for diagnostic purposes on a three-stage scale (good, acceptable, and poor). Before the experiment started, each observer attended an introductory session in which several instructional cases were evaluated.

Conventional ROC curves for each observers were obtained by comparing the presence or absence of abnormalities with the observer's degree of certainty. For a comparison of paired ROC data, the area under each ROC curve

Table 3. Comparison of Areas Under the ROC Curves Between Film and CRT in Each Group Comprising Six Observers

\begin{tabular}{|c|c|c|c|c|c|c|c|c|}
\hline & \multicolumn{2}{|c|}{ Group A } & \multicolumn{2}{|c|}{ Group B } & \multicolumn{2}{|c|}{ Group C } & \multicolumn{2}{|c|}{ Group D } \\
\hline & NGY Exp. 1 & MYD Exp. 2 & MYD Exp. 1 & NGY Exp. 2 & NGY Exp. 1 & NGY Exp. 2 & MYD Exp. 1 & MYD Exp. 2 \\
\hline Film & $0.877(0.02)$ & $0.857(0.03)$ & $0.716(0.03)$ & $0.835(0.03)$ & $0.793(0.03)$ & $0.866(0.03)$ & $0.815(0.03)$ & $0.833(0.03)$ \\
\hline CRT & $0.873(0.02)$ & $0.851(0.03)$ & $0.787(0.03)$ & $0.801(0.03)$ & $0.785(0.03)$ & $0.836(0.03)$ & $0.798(0.03)$ & $0.841<0.03\}$ \\
\hline
\end{tabular}

Values in parentheses show standard error. 
Table 5. Comparison of Areas Under the ROC Curves Between Film and CRT in the Total of 24 Observers

\begin{tabular}{cccc}
\hline & $\begin{array}{c}\text { NGY and MYD: } \\
\text { Exp. } 1\end{array}$ & $\begin{array}{c}\text { NGY and MYD: } \\
\text { Exp. 2 }\end{array}$ & Total \\
\hline Film & $0.801(0.01)$ & $0.851(0.01)$ & $0.826(0.01)$ \\
CRT & $0.810(0.01)$ & $0.835(0.01)$ & $0.823(0.01)$ \\
\hline
\end{tabular}

Values in parentheses show standard error.

was calculated from each binormal ROC curve, which was fit to the observer's confidence rating data by means of a maximum likelihood estimation. ${ }^{20,21}$ Statistical evaluation was performed using the method described by Hanley and McNeil. ${ }^{22}$ Average ROC curves were obtained by averaging the binormal slope and intercept parameters of the individual observer's ROCs. For calculation of sensitivity and specificity, confidence scores of 2 and 4 were included with correct answers, and the confidence score of 3 was grouped with incorrect answers. Significance was evaluated by twoway analysis of variance. ${ }^{23}$

\section{RESULTS}

\section{ROC Analysis}

Comparison between film and CRT images by individual observers showed no significant differences between film and CRT images on ROC analysis. There was no deference in performance between the two kinds of CRT monitor images used by individual observers in the facility-alternated group.

In comparison of film reading with CRT reading in each group, there were no significant differences (Table 3 ). With respect to the overall total of 12 observers at both facilities (Table 4), the overall total of 24 observers at the two facilities for the first and second experiments, and the total combined results (Table 5), film and CRT reading were compared, but there were no differences detected. Moreover, no significant differences were observed between the two kinds of CRTs in groups A and B (Table 3).

In comparing the order of observation during the 2-day sessions at the two facilities in the first and second experiments, no significant differ-
Table 7. Comparison of Areas Under the ROC Curves Between Film and CRT With Regard to Observer's Experience

\begin{tabular}{cccccc}
\hline & \multicolumn{4}{c}{ 5 Observers } \\
\cline { 2 - 3 } \cline { 5 - 6 } & \multicolumn{2}{c}{ Exp. 1 } & Exp. 2 & & \multicolumn{2}{c}{$<$ Exp years 1} & Exp. 2 \\
\hline Film & $0.859(0.03)$ & $0.888(0.03)$ & $0.807(0.03)$ & $0.941(0.02)$ \\
CRT & $0.871(0.03)$ & $0.855(0.03)$ & $0.850(0.03)$ & $0.869(0.03)$
\end{tabular}

Values in parentheses show standard error.

ences were found between film and CRT reading (Table 6).

A group of five observers who were experienced chest radiologists with more than 10 years experience in reading chest radiographs as well as a group of five observers with less experience in reading chest radiographs (less than 5 years) were selected from among the observers, and comparison between films and CRT images was performed for each of these groups. The two groups showed no significant difference in performance (Table 7).

\section{Diagnostic Efficacy in Each Disease Category}

Diagnostic efficacy of uncompressed original images was evaluated in the first experiment according to each disease category, ie, normal $(\mathrm{n}=18)$, interstitial disease including granular and linear shadows $(n=11)$, nodular shadow between 11 and $30 \mathrm{~mm}$ in size $(\mathrm{n}=11)$, and 10 $\mathrm{mm}$ and below in size $(\mathrm{n}=24)$ except 7 cases not included in such categories. There was no significant difference between film and CRT in specificity and sensitivity in detecting pulmonary nodules. However, in detecting interstitial abnormalities, although the sensitivity for both techniques was low, CR film was significantly more sensitive than CR CRT (Table 8).

Comparison of Diagnostic Efficacy Between Original Images in the First Experiment and Compressed Images in the Second Experiment

No statistically significant differences between original images and irreversibly com-

Table 6. Comparison of Areas Under the ROC Curves Between Film and CRT With Regard to the Order of Interpretation During 2-Day Sessions at Two Facilities

\begin{tabular}{|c|c|c|c|c|c|c|c|c|}
\hline & \multicolumn{4}{|c|}{ NGY } & \multicolumn{4}{|c|}{ MYD } \\
\hline & \multicolumn{2}{|c|}{$\mathrm{CRT} \rightarrow$ FILM } & \multicolumn{2}{|c|}{ FILM $\rightarrow$ CRT } & \multicolumn{2}{|c|}{$\mathrm{CRT} \rightarrow \mathrm{FILM}$} & \multicolumn{2}{|c|}{$\mathrm{FILM} \rightarrow \mathrm{CRT}$} \\
\hline & Exp. 1 & Exp. 2 & Exp. 1 & Exp. 2 & Exp. 1 & Exp. 2 & Exp. 1 & Exp. 2 \\
\hline Film & $0.887(0.03)$ & $0.877(0.03)$ & $0.790\{0.03\}$ & $0.820(0.03)$ & $0.803(0.03)$ & $0.790(0.03)$ & $0.726(0.03)$ & $0.893(0.02)$ \\
\hline CRT & $0.851(0.03)$ & $0.843(0.03)$ & $0.812\langle 0.03\rangle$ & $0.802(0.03)$ & $0.846(0.03)$ & $0.824(0.03)$ & $0.737(0.03)$ & $0.866(0.03)$ \\
\hline
\end{tabular}

Values in parentheses show standard error. 
Table 8. Comparison of Diagnostic Efficacy Between Film and CRT in Exp. 1

\begin{tabular}{|c|c|c|c|c|}
\hline & \multirow{2}{*}{$\begin{array}{c}\text { Specificity } \\
\text { Normal } \\
\text { Case } \\
(n=432)\end{array}$} & \multicolumn{3}{|c|}{ Sensitivity } \\
\hline & & $\begin{array}{c}\text { Interstitial } \\
\text { Disease } \\
(n=264)\end{array}$ & $\begin{array}{l}\geq 10 \mathrm{~mm} \\
\text { Nodule } \\
(\mathrm{n}=264)\end{array}$ & $\begin{array}{c}11-30 \mathrm{~mm} \\
\text { Nodule } \\
(\mathrm{n}=576)\end{array}$ \\
\hline Film & 69.6 & $58.3^{*}$ & 57.6 & 84.4 \\
\hline CRT & 72.6 & $49.7^{*}$ & 56.4 & 85.8 \\
\hline
\end{tabular}

${ }^{*} P<.05$.

pressed images were found both for film and CRT (Tables 9 and 10).

\section{Analysis of Image Quality}

Image quality of 45 cases irreversibly compressed in the second experiment were compared with those not compressed in the first experiment by subjective evaluation in three stages. The results showed that for both film and CRT images, a high proportion of $10: 1$ and 20:1 compressed images were judged to be poor for clinical use, and significant differences from the original images were recognized (Table 11).

\section{DISCUSSION}

One of the final goals of the PACS is to use soft-copy display of various kinds of diagnostic imaging for primary interpretation by radiologists. CR is a suitable and important digital technique for implementation of PACS because plain radiograph and other projection radiographs are the most frequently obtained images in the diagnostic radiology department. The success of implementation of PACS is highly dependent on the functionality and performance of the viewing workstation. With regard to the comparison of film and CRT displays of CR images, reports have previously appeared concerning the capability for delineation of fine structure and lesions. ${ }^{13,14}$ Elaim et al ${ }^{11}$ concluded that radiologists interpreting digital workstation images $(1,024 \times 1,536$-pixel monitor) were significantly less sensitive for detecting pneumothoraces than film-screen and small format $\mathrm{CR}$ images, although no significant differences were found in the area under ROC curves. Razavi et a ${ }^{15}$ concluded that chest CR images in children viewed in soft-copy PACS environment $(2,048-\times 2,048$-pixel monitor $)$ resulted in diagnoses similar to or slightly more accurate than those obtained in a laser-printed film-based environment. Frank et $\mathrm{al}^{16}$ documented that a CRT display system $(2,048-\times 2,560$ line monitor) can provide diagnostic information effectively equal to CR film for the abnormalities studied if the CRT image is displayed with spatial resolution compatible to hard copy and contrast resolution is sufficiently optimized on the CRT system. These results indicate that differences in the spatial resolution of digital workstation may have affected the sensitivity in detecting subtle abnormalities.

A carefully designed ROC study is an ideal method for analyzing the spatial requirement for diagnostic radiographs. In our ROC study, interstitial shadows and pneumothorax were chosen as a measure of the spatial-resolution requirement. Nodules were chosen as a test of contrast resolution because their detection depends on contrast with the background. Differences among observers and the number of observers also may affect the results of an ROC study. Not only studies within a single institution with a few observers, but also large-scale cooperative experimental studies are necessary to more thoroughly evaluate the possibilities CRT diagnosis. Thus, in the present study, large-scale reading tests involving multiple observers from several medical institutions were conducted to ameliorate such differences in the pooled results.

In evaluating the film and CRT images, because the same images were observed in both film and CRT form for 2 consecutive days during the first and second experiments, a

Table 9. Comparison of Diagnostic Efficacy Between Original and Compression at 10:1

\begin{tabular}{|c|c|c|c|c|c|c|c|c|}
\hline & \multirow{2}{*}{\multicolumn{2}{|c|}{$\begin{array}{c}\text { Specificity } \\
\text { Normal } \\
(n=168)\end{array}$}} & \multicolumn{6}{|c|}{ Sensitivity } \\
\hline & & & \multicolumn{2}{|c|}{$\begin{array}{l}\text { Interstitial Disease } \\
\qquad(n=144)\end{array}$} & \multicolumn{2}{|c|}{$\begin{array}{c}10 \mathrm{~mm} \geq \text { Nodule } \\
(\mathrm{n}=120)\end{array}$} & \multicolumn{2}{|c|}{$\begin{array}{c}11-30 \mathrm{~mm} \text { Nodule } \\
(\mathrm{n}=144)\end{array}$} \\
\hline & $1: 1$ & $10: 1$ & $1: 1$ & $10: 1$ & $1: 1$ & $10: 1$ & $1: 1$ & 10:1 \\
\hline Film & 75.6 & 83.9 & 68.8 & 78.5 & 44.2 & 51.7 & 84.7 & 86.8 \\
\hline CRT & 75.0 & 78.6 & 59.0 & 69.4 & 43.3 & 49.2 & 86.8 & 87.5 \\
\hline
\end{tabular}

$1: 1$ signifies original without compression. 
Table 10. Comparison of Diagnostic Efficacy Between Original and Compression at 20:1

\begin{tabular}{|c|c|c|c|c|c|c|c|c|}
\hline & \multirow{2}{*}{\multicolumn{2}{|c|}{$\begin{array}{c}\text { Specificity } \\
\begin{array}{c}\text { Normal } \\
(\mathrm{n}=72)\end{array}\end{array}$}} & \multicolumn{6}{|c|}{ Sensitivity } \\
\hline & & & \multicolumn{2}{|c|}{$\begin{array}{l}\text { Interstitial Disease } \\
\qquad(\mathrm{n}=72)\end{array}$} & \multicolumn{2}{|c|}{$\begin{aligned} & 10 \mathrm{~mm} \geq \text { Nodule } \\
&(\mathrm{n}=48)\end{aligned}$} & \multicolumn{2}{|c|}{$\begin{array}{c}11-30 \mathrm{~mm} \text { Nodule } \\
(\mathrm{n}=240)\end{array}$} \\
\hline & $1: 1$ & $20: 1$ & $1: 1$ & $20: 1$ & $1: 1$ & $20: 1$ & $1: 1$ & $20: 1$ \\
\hline Film & 77.8 & 79.1 & 55.6 & 51.4 & 52.1 & 37.5 & 87.5 & 92.2 \\
\hline CRT & 77.8 & 81.9 & 44.4 & 50.0 & 62.5 & 50.0 & 91.7 & 90.1 \\
\hline
\end{tabular}

$1: 1$ signifies original without compression.

question arose as to whether the memory of the images viewed on the previous day might affect the results of readings on the subsequent day. Accordingly, a group such that the image was first viewed as film and a group such that the image was first viewed on a CRT were distinguished, and the comparison showed no significant differences. Thus, the influence of this factor upon the analytical results appears to be slight. In the present study, comparison by the individual readers showed no significant differences between the diagnostic results obtained from films and CRT displays. The low sensitivity for both film and CRT monitor in detecting interstitial disease indicated that some cases were too severe to detect minor differences. Although analyses of the area under the ROC curves showed no significant differences in detection of pulmonary abnormalities between film and CRT, lower sensitivity in detecting interstitial abnormalities on CRT than on film shown in this study may have been affected by the lower resolution of the CRT workstation in comparison to that with a $2 \mathrm{~K}-\times 2 \mathrm{~K}$-pixel matrix, as well as observers' unfamiliarity with the CRT workstation ${ }^{11}$ and uneffective utilization image processing including zooming procedure and high-frequency edge-enhanced processing. ${ }^{24}$ Further consideration with regard to spatial resolution requirements and image processing is needed.

Regarding image compression, the proportion of subjective judgments indicating that even 10:1 compressed images were poor as chest radiographs was significantly higher than that of uncompressed images. However, there were no differences clearly associated with the compression ratio noted in diagnostic accuracy. The small number of cases in the respective groups may be one of the causes of this discrepancy. In addition, another reason for the discrepancy was the fact that diagnostic accuracy does not depend on spatial resolution alone (visibility of fine objects, such as the thin linear shadow of pneumothorax), but also on the categories of objects and other factors: contrast, density, size, and shape of objects. Previously reported studies of irreversible compression by ROC analysis have indicated that 10:1 compression is clinically suitable for detection of lesions on CR chest radiographs. ${ }^{17}$ Nevertheless, further studies are necessary to ascertain whether diagnostic utility is affected.

\section{CONCLUSION}

Film and CRT displays of 71 plain CR chest radiographs were each interpreted twice by 20 radiologists and four chest internists specializing in respiratory disease. The following conclusions were obtained. (1) Analyses of the areas under the ROC curves showed no statistically significant differences in detection of pulmonary abnormalities between film and CRT. However, in detecting interstitial pulmonary abnormalities, film was more sensitive than the CRT monitor. (2) No differences in observers' performance were found between two different kinds of CRT workstation. (3) Subjective evaluation

Table 11. Subjective Evaluation of Image Quality Between Original and Compression

\begin{tabular}{|c|c|c|c|c|c|c|c|c|}
\hline & \multicolumn{2}{|c|}{ FILM } & \multicolumn{2}{|c|}{ CRT } & \multicolumn{2}{|c|}{ FILM } & \multicolumn{2}{|c|}{ CRT } \\
\hline & $1: 1$ & $10: 1$ & $1: 1$ & $10: 1$ & $1: 1$ & $20: 1$ & $1: 1$ & $20: 1$ \\
\hline Good & 51.9 & 45.8 & 47.0 & 29.7 & 50.2 & 43.9 & 46.3 & 30.4 \\
\hline Acceptable & 44.6 & 47.9 & 49.0 & 58.7 & 45.2 & 49.3 & 48.0 & 59.7 \\
\hline Poor & $3.5^{*}$ & $6.3^{*}$ & $4.0 \dagger$ & $11.6 t$ & 4.6 & 6.8 & $5.7 \dagger$ & $9.9 \dagger$ \\
\hline
\end{tabular}

$* P<.05$.

$+P<.01$. 
of image quality showed that images irreversibly compressed to the ratios of $10: 1$ and $20: 1$ were inferior to uncompressed images. (4) The utilization of CR CRT image as a substitute for CR film is possible. From the viewpoint of clinical acceptability, further considerations are needed with regard to resolution requirements, image processing, and image compression.

\section{REFERENCES}

1. Sonoda M, Takano M, Miyahara J, et al: Computed radiography utilizing scanning laser stimulated luminescence. Radiology 148:833-838, 1983

2. Foley WD, Wilson CR, Keyes GS, et al: The effect of varying spatial resolution on the detectability of diffuse pulmonary nodules. Assessment with digitized conventional radiography. Radiology 141:25-31, 1981

3. Lams PM, Cocklin ML: Spatial resolution requirements for digital chest radiographs: An ROC study of observer performance in selected cases. Radiology 158:1119, 1986

4. McMahon H, Vyborny $\mathrm{CJ}$, Metz CE, et al: Digital radiography of subtle pulmonary abnormalities: An ROC study of the effect of pixel size on observer performance. Radiology 158:21-26, 1986

5. Goodman LR, Foley WD, Wilson CR, et al: Digital and conventional chest images: Observer performance with film digital radiography system. Radiology 158:27-33, 1986

6. Kastan DJ, Ackerman LV, Feczko PJ: Digital gastrointestinal imaging: The effect of pixel size on detection of subtle mucosal abnormalities. Radiology 162:853-856, 1987

7. Seely GW, Fisher HD, Stempski MO, et al: Total digital radiology department: Spatial resolution requirement. AJR 148:421-426, 1987

8. Hayrapetian A, Aberle DR, Huang HK, Fiske R, et al: Comparison of 2048-line digital display formats and conventional radiographs: An ROC study. AJR 152:1113-1118, 1989

9. Franken EA Jr, Berbaum KS, Marley SM, et al Evaluation of a digital workstation for interpreting neonatal examinations. A receiver operating characteristic study. Invest Radiol 27:732-737, 1992

10. Slasky BS, Gur D, Good WF, et al: Receiver operating characteristic analysis of chest imaging interpretation with conventional, laser-printed, and high-resolution workstation images. Radiology 174:775-780, 1990

11. Elam EA, Rehm K, Hillman BJ, et al: Efficacy of digital radiography for the detection of pneumothorax: Comparison with conventional chest radiography. AJR 158:509-514, 1992

12. Ackerman SJ, Gitlin JN, Gayler RW, et al: Receiver operating characteristic analysis of fracture and pneumonia detection: Comparison of laser-digitized workstation images and conventional analog radiographs. Radiology 186: 263-268, 1993

13. Itoh $\mathrm{Y}$, Ishigaki $\mathrm{T}$, Sakuma $\mathrm{S}$ : Evaluation of digital radiographs on CRT displays-A basic and clinical study. Nippon Acta Radiol (J Jpn Radiol Soc) 48:1024-1031, 1988

14. Ishigaki T, Sakuma S, Maruyama $K$, et al: CRT images of projection radiographs: Comparison with film images. Med Imaging Technol 7:334-343, 1989

15. Razavi M, Sayre JW, Taira RK, et al: Receiveroperating-characteristic study of chest radiographs in children: Digital hard-copy film vs $2 \mathbf{k} \times 2 \mathbf{k}$ soft-copy images. AJR 158:443-448, 1992

16. Frank MS, Jost RG, Molina PL, et al: Highresolution computer display of portable, digital, chest radiographs of adults: Suitability for primary interpretation. AJR 160:473-477, 1993

17. Ishigaki $T$, Sakuma $S$, Ikeda $M$, et al: Clinical evaluation of irreversible image compression: Analysis of chest imaging with computed radiography. Radiology 175 : 739-743, 1990

18. Shimura K, Ishida M, Kato H: Study on new data compression method for CR image. Jpn J Radiol Technol 43:997, 1991 (abst)

19. Itoh Y, Ishigaki T, Sakuma S, et al: Influence of CRT workstation on observer's performance. Comput Methods Programs Biomed 37:253-258, 1992

20. Dolfman DD, Alf E: Maximum likelihood estimation of parameters of signal detection theory and determination of confidence intervals: Rating method data. J Math Psych 6:487-496, 1969

21. Mets CR: ROC methodology in radiological imaging. Invest Radiol 21:720-733, 1986

22. Hanley JA, McNeil BJ: A method of comparing the area under receiver operating characteristic curves derived from the same cases. Radiology 148:839-843, 1983

23. Kleinbaum DG, Kupper LL: Applied regression analysis and other multivariable methods. Boston, MA, Duxbury, 1978, pp 315-375

24. Shaefer CM, Greene R, Llewellyn HJ, et al: Interstitial lung disease: Impact of postprocessing in digital storage phosphor imaging. Radiology 178:733-738, 1991 